\title{
Measurement of Intrahepatic Pressure during Microwave Ablation in an Ex Vivo Bovine Liver Model
}

\author{
Hae Jin Kim, Hyunchul Rhim, Min Woo Lee, and Woo Kyoung Jeong \\ Department of Radiology and Center for Imaging Science, Samsung Medical Center, Sungkyunkwan University School of Medicine, Seoul, \\ Korea
}

Background/Aims: We experimented with different ablation methods and two types of microwave antennas to determine whether microwave ablation (MWA) increases intrahepatic pressure and to identify an MWA protocol that avoids increasing intrahepatic pressure. Methods: MWA was performed using either a single-step standard ablation or a stepwise increment ablation paired with either a 16-gauge (G) 2-cm antenna or a $14 \mathrm{G} 4-\mathrm{cm}$ antenna. We compared the maximum pressures and total ablation volumes. Results: The mean maximum intrahepatic pressures and ablation volumes were as follows: $16 \mathrm{G}$ single-step: $37 \pm 33.4 \mathrm{~mm} \mathrm{Hg}$ and $4.63 \mathrm{~cm}^{3}$; $16 \mathrm{G}$ multistep: $31 \pm 18.7 \mathrm{~mm} \mathrm{Hg}$ and $3.75 \mathrm{~cm}^{3}$; $14 \mathrm{G}$ singlestep: $114 \pm 45.4 \mathrm{~mm} \mathrm{Hg}$ and $15.33 \mathrm{~cm}^{3}$; and $14 \mathrm{G}$ multistep: $106 \pm 43.8 \mathrm{~mm} \mathrm{Hg}$ and $10.98 \mathrm{~cm}^{3}$. The intrahepatic pressure rose during MWA, but there were no statistically significant differences between the single and multistep methods when the same gauge antennae were used. The total ablation volume was different only in the $14 \mathrm{G}$ groups $(p<0.05)$. Conclusions: We demonstrated an increase in intrahepatic pressure during MWA. The multistep method may be used to prevent increased intrahepatic pressure after applying the proper power. (Gut Liver 2015;9:784-790)

Key Words: Microwave ablation; Intrahepatic pressure; Ex vivo; Liver

\section{INTRODUCTION}

Radiofrequency ablation (RFA) has been accepted clinically as a useful local treatment for hepatocellular carcinoma (HCC). ${ }^{1,2}$ But intrahepatic dissemination, ${ }^{3,4}$ extrahepatic seeding, ${ }^{5}$ and rapidly growing recurrences ${ }^{6,7}$ have recently been reported.

Several reports suggested that these recurrences might be at- tributed to increased intratumoral pressure. Portolani et al. ${ }^{8}$ documented four cases of aggressive recurrence after RFA of liver tumors. Seki et al. ${ }^{9}$ have also reported a case with rapid progression of numerous tumors around the ablation zone after RFA. They suggested that an increase in local pressure induced by RFA caused the unwanted tumor dissemination. They hypothesize that RFA during energy application may increase intratumoral pressure, aggravating intravascular spread of the tumor to the surrounding tissues. Kotoh et al. ${ }^{10,11}$ subsequently reported that liver parenchymal pressure increased during RFA, and they developed a multistep incremental expansion method for RFA in vitro and in vivo that prevented increases in pressure. Kawamoto et al. ${ }^{12}$ reported that hepatic parenchymal pressure increased during RFA, and suggested that rapid tumor heating may lead to an unpredicted increase in intratumoral pressure. However, increased intrahepatic pressure can be controlled using multistep methods. In particular, LeVeen needle multistep methods should be used to prevent increased intrahepatic pressure during RFA.

Microwave ablation (MWA) is the recent development in the field of tumor ablation ${ }^{7,13-15}$ and is expected to be as effective as RFA for local treatment. Microwave energy is found along the spectrum of electromagnetic radiation, heating the target tissue. Electromagnetic microwaves heat matter by agitating water molecules in the adjacent tissue, producing friction and heat and therefore inducing cellular death via coagulation necrosis. ${ }^{16}$ Microwave energy has several benefits over radiofrequency energy for tumor ablation, and it has been accepted as a useful local treatment for thermal coagulation. ${ }^{16-18}$ However, intratumoral pressure is expected to rise during MWA because microwave energy uses heating to achieve its aims, alike RFA. ${ }^{3}$

No studies exist on MWA-induced increased intrahepatic pressure and the potential for an associated increase in intratu-

Correspondence to: Hyunchul Rhim

Department of Radiology, Samsung Medical Center, Sungkyunkwan University School of Medicine, 81 Irwon-ro, Gangnam-gu, Seoul 06351, Korea Tel: +82-2-3410-2507, Fax: +82-2-3410-6368, E-mail: rhimhc@skku.edu

Received on July 18, 2014. Revised on October 25, 2014. Accepted on November 7, 2014. Published online May 13, 2015 pISSN 1976-2283 eISSN 2005-1212 http://dx.doi.org/10.5009/gnl14272

() This is an Open Access article distributed under the terms of the Creative Commons Attribution Non-Commercial License (http://creativecommons.org/licenses/by-nc/4.0) which permits unrestricted non-commercial use, distribution, and reproduction in any medium, provided the original work is properly cited. 
moral pressure during MWA. Variety of conditions cause pressure to rise and its relation to ablation volume such as type and length of antenna, power, or duration of ablation time. We hypothesize that during MWA, intrahepatic pressure arises, but can be controlled using multistep methods. ${ }^{11}$ Significant research is required to determine if MWA, like RFA, increases intratumoral pressure. Therefore, we used ex vivo experiments to determine whether intrahepatic pressure rises during MWA, which conditions affect the pressure rise, and how such conditions influence the ablation volume.

\section{MATERIALS AND METHODS}

\section{Study design}

EX-vivo bovine liver model was used as a model of a HCC tumor. To mimic the temperature of the human body, we soaked a block of cow liver in a water tank and heated it wrapped in a plastic case in $40^{\circ} \mathrm{C}$ water until just before the start of the ex- periment about 30 minutes. There was about 7 to 8 hours time interval between organ harvest and experiment.

After placing a heated cow liver on a flat plate, we inserted a microwave antenna (MedWaves, San Diego, CA, USA) (Fig. 1) and pressure sensing needle (STARmed, Seoul, Korea) (Fig. 2) perpendicularly into the cow liver. A 16-gauge (G) antenna with a 2-cm active tip and a $14 \mathrm{G}$ antenna with a 4-cm tip were used. The tips of the antennae and pressure sensing needle were fixed at the proximal portion (Fig. 3). The width between the two tips was maintained within 1 to $2 \mathrm{~mm}$. The average depth of insertion was $6 \mathrm{~cm}$ for the $14 \mathrm{G}$ antenna and $4 \mathrm{~cm}$ for the $16 \mathrm{G}$ antenna.

The microwave antenna was connected to a microwave generator (AveCure Microwave Generator; MedWaves) (Fig. 1). This microwave generator uses a dynamic frequency range (902 to $908 \mathrm{MHz}$ ) capable of adapting to tissue permittivity changes during ablation. It can monitor power levels automatically and adjusts frequency to maximize power delivery to the targeted
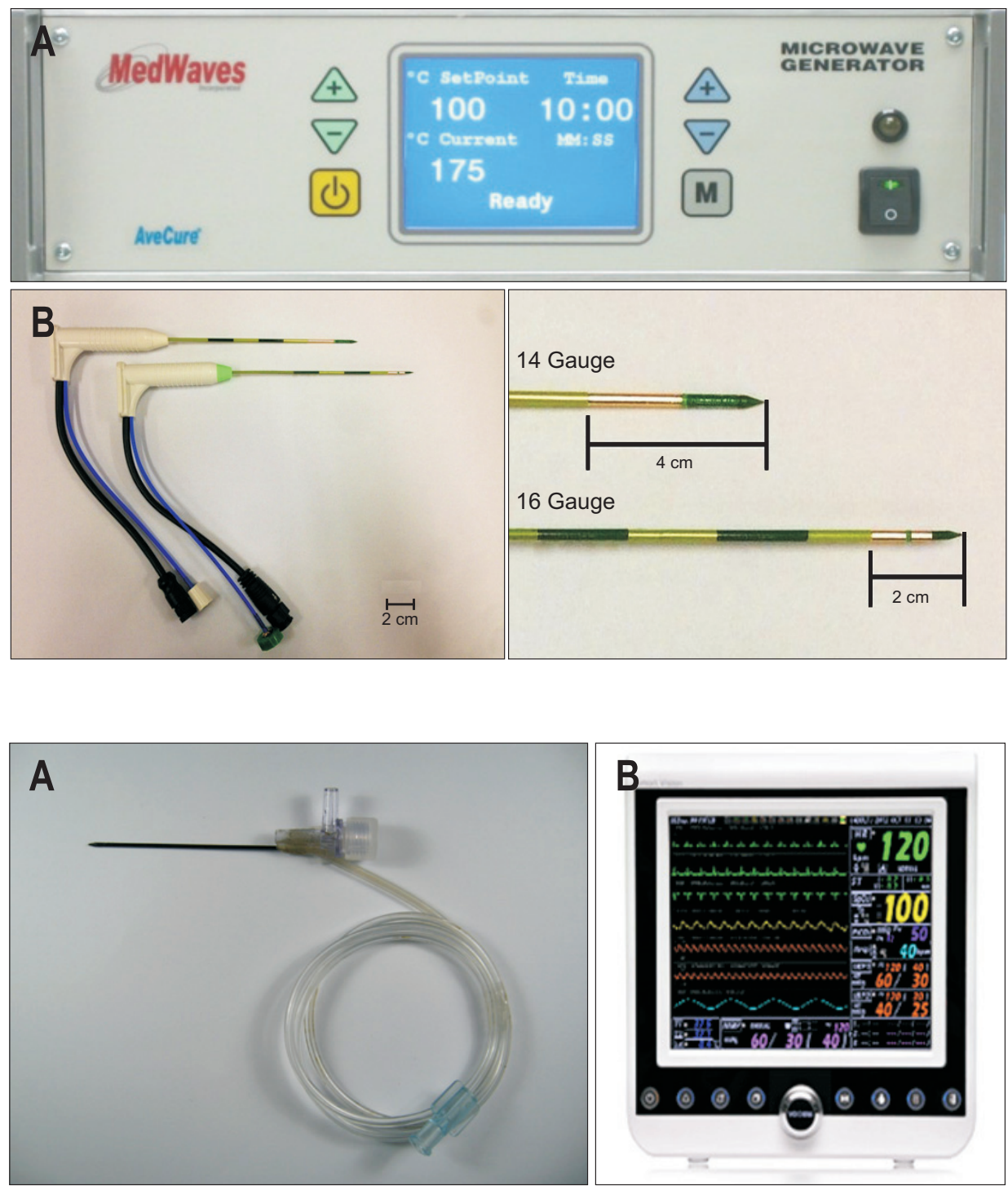

Fig. 1. Microwave generator and needle antenna (AveCure Microwave Generator; MedWaves). (A) We used a microwave generator to create a dynamic frequency range (902 to $908 \mathrm{MHz}$ ) capable of adapting to tissue permittivity changes during ablation. It monitors power levels automatically and adjusts the frequency to maximize power delivery to the targeted tissue. (B) A 2-cm active tip with a 16-gauge (G) (lower figure) antenna and a 4-cm tip with a $14 \mathrm{G}$ antenna (upper figure) were used.

Fig. 2. Pressure sensing needle (STARmed) and pressure monitoring device (VP-1000; VOTEM Co., Ltd.). (A) A percutaneous pressure-sensing needle was inserted into the cow liver. The pressure rise in the cow liver can be determined by measuring the pressure of saline in the transparent line, which is connected to the pressure monitoring device. (B) Pressure monitoring device (VP-1000). 
tissue.

We monitored intrahepatic pressure using a $19 \mathrm{G}$ pressure sensing needle $(1.06 \mathrm{~mm})$ connected to a pressure measuring machine (VP-1000; VOTEM Co., Ltd., Seoul, Korea) (Fig. 2).

\section{MWA protocol}

MWA was performed using standard single-step ablation with a16G antenna, stepwise multistep ablation with a 16G antenna, standard single-step ablation with a $14 \mathrm{G}$ antenna, and stepwise multistep ablation with a $14 \mathrm{G}$ antenna. Ten pieces of cow liver

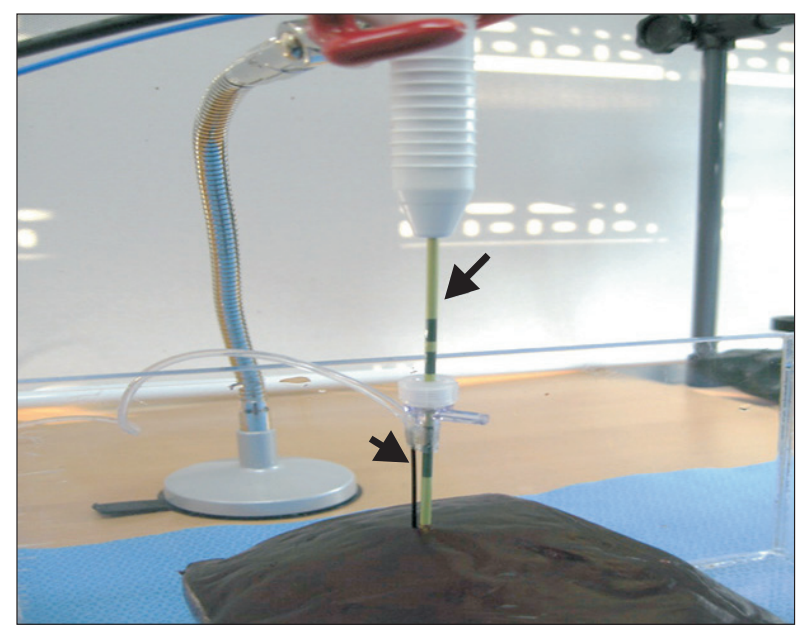

Fig. 3. Setting of microwave antenna and pressure-sensing needle. The microwave antenna (long arrow) and the pressure-sensing needle (short arrow) were perpendicularly inserted into the liver, parallel with one another. These two tips were fixed and immobilized around the hub. The width between the two tips was maintained between 1 and $2 \mathrm{~mm}$. The antenna tip was located $6 \mathrm{~cm}$ from the liver surface for the 14-gauge $(\mathrm{G})$ antenna and $4 \mathrm{~cm}$ for the $16 \mathrm{G}$ antenna. were used in each group and 40 pieces were used in total. The maximum intrahepatic pressure at each pulsing were measured during the ablation process and were recorded.

In the group with standard single-step ablation with a $16 \mathrm{G}$ antenna $(\mathrm{n}=10)$, the power was applied at $24 \mathrm{~W}$. In the group that received stepwise multistep ablation with a $16 \mathrm{G}$ antenna $(\mathrm{n}=10)$, the power was gradually increased from $14 \mathrm{~W}$ to $16 \mathrm{~W}$, $18 \mathrm{~W}, 20 \mathrm{~W}$, and finally $24 \mathrm{~W}$ at 1 minute intervals (Table 1 ).

In the group that received standard single-step ablation with a $14 \mathrm{G}$ antenna $(\mathrm{n}=10)$, the power was applied at $28 \mathrm{~W}$ and maintained. In the stepwise multistep ablation with a $14 \mathrm{G}$ antenna $(\mathrm{n}=10)$, the power was gradually increased from $16 \mathrm{~W}$ to $18 \mathrm{~W}, 20 \mathrm{~W}, 24 \mathrm{~W}$, and finally $28 \mathrm{~W}$ at 1 minute intervals (Table 1). MWA protocol was different between $16 \mathrm{G}$ antenna and $14 \mathrm{G}$ antenna. Start watt should be same but we followed optimized condition in each antenna which was proposed by manufacturer.

\section{Measurement of volume of ablation zone}

To reduce bias, we used a method that measured the distance through the photo taken of the specimens. We measured the length and depth of a longitudinal section and the length and width of a cross section. We then transformed the real numeric value using a survey program (Image J program). Ablation volume was calculated by following equation:

Depth of a longitudinal section $\times$ short distance of cross section $\times$ long distance of cross section $\times \pi / 6$

\section{Statistical analysis}

Maximum intrahepatic pressures obtained under each MWA protocol and ablation volume were compared using the KruskalWallis test and Tukey test using ranks. A p-value lower than 0.05

Table 1. Four Protocols for Microwave Ablation

\begin{tabular}{clcl}
\hline Electrode & Step & Start watt, W & \multicolumn{1}{c}{ Condition } \\
\hline 16G antenna & Single & 24 & Maintain 24 W \\
& Multi & 14 & 5 Steps with14, 16, 18, 20, 24 W \\
& Single & 28 & Maintain 28 W \\
& Multi & 16 & 5 Steps with 16, 18, 20, 24, 28 W \\
\hline
\end{tabular}

G, gauge; W, watt.

Table 2. Maximum Pressure and Mean Ablation Volume of the Four Groups

\begin{tabular}{|c|c|c|c|c|c|c|c|c|c|c|}
\hline \multirow{2}{*}{ Electrode } & \multirow{2}{*}{ Step } & \multirow{2}{*}{ Case } & \multirow{2}{*}{$\begin{array}{l}\text { Temperature } \\
\text { of the liver, }{ }^{\circ} \mathrm{C}\end{array}$} & \multirow{2}{*}{$\begin{array}{c}\text { Maximal } \\
\text { pressure, } \\
\text { mm Hg }\end{array}$} & \multicolumn{2}{|c|}{ Longitudinal section, cm } & \multicolumn{2}{|c|}{ Cross section, $\mathrm{cm}$} & \multirow{2}{*}{$\begin{array}{c}\text { Mean } \\
\text { ablation } \\
\text { volume, } \mathrm{cm}^{3}\end{array}$} & \multirow{2}{*}{$\begin{array}{l}\text { Ablation time, } \\
\text { sec }\end{array}$} \\
\hline & & & & & Width & Depth & Short axis & Long axis & & \\
\hline \multirow[t]{2}{*}{$16 \mathrm{G}$} & Single & 10 & 19.0 & $37 \pm 33.4$ & 1.74 & 2.81 & 1.72 & 1.84 & $4.63 \pm 0.5$ & 675 \\
\hline & Multi & 10 & 18.2 & $31 \pm 18.7$ & 1.69 & 2.70 & 1.56 & 1.69 & $3.75 \pm 0.8$ & 401 \\
\hline \multirow[t]{2}{*}{$14 \mathrm{G}$} & Single & 10 & 19.7 & $114 \pm 45.4$ & 2.23 & 4.99 & 2.21 & 2.38 & $15.33 \pm 3.4$ & 339 \\
\hline & Multi & 10 & 19.0 & $106 \pm 43.8$ & 1.96 & 4.34 & 1.92 & 2.14 & $10.98 \pm 2.5$ & 306 \\
\hline
\end{tabular}

$\mathrm{G}$, gauge. 
was considered statistically significant.

\section{RESULTS}

The maximal intrahepatic pressure and mean ablation volume in each group are summarized in Table 2. We also presented peak pressure measurement of all 40 data in Table 3 and Fig. 4. Average temperature of cow liver tissue was $18^{\circ} \mathrm{C}$ which had discrepancy of water temperature.

\section{Intrahepatic pressure}

The average maximum intrahepatic pressure was as follows: standard single-step ablation with $16 \mathrm{G}$ antenna, $37 \pm 33.4 \mathrm{~mm} \mathrm{Hg}$; stepwise multistep ablation with $16 \mathrm{G}$ antenna, $31 \pm 18.7 \mathrm{~mm} \mathrm{Hg}$; standard single-step ablation with $14 \mathrm{G}$ antenna, $114 \pm 45.4 \mathrm{~mm} \mathrm{Hg}$;

Table 3. Peak Pressure Measurement of All 40 Data Measures per Group

\begin{tabular}{|c|c|c|c|c|}
\hline & \multicolumn{2}{|c|}{$16 \mathrm{G}$} & \multicolumn{2}{|c|}{$14 \mathrm{G}$} \\
\hline & Single & Multi & Single & Multi \\
\hline \multirow{10}{*}{$\begin{array}{l}\text { Maximal pressure } \\
\text { per case, } \mathrm{mm} \mathrm{Hg}\end{array}$} & 13 & 21 & 140 & 137 \\
\hline & 33 & 32 & 129 & 126 \\
\hline & 104 & 56 & 139 & 100 \\
\hline & 89 & 63 & 84 & 75 \\
\hline & 13 & 17 & 192 & 122 \\
\hline & 20 & 38 & 61 & 100 \\
\hline & 23 & 23 & 55 & 107 \\
\hline & 47 & 43 & 96 & 141 \\
\hline & 14 & 13 & 79 & 77 \\
\hline & 13 & 6 & 161 & 164 \\
\hline Average \pm SD & $37 \pm 33.4$ & $31 \pm 18.7$ & $114 \pm 45.4$ & $106 \pm 43.8$ \\
\hline
\end{tabular}

$\mathrm{G}$, gauge; $\mathrm{SD}$, standard deviation.

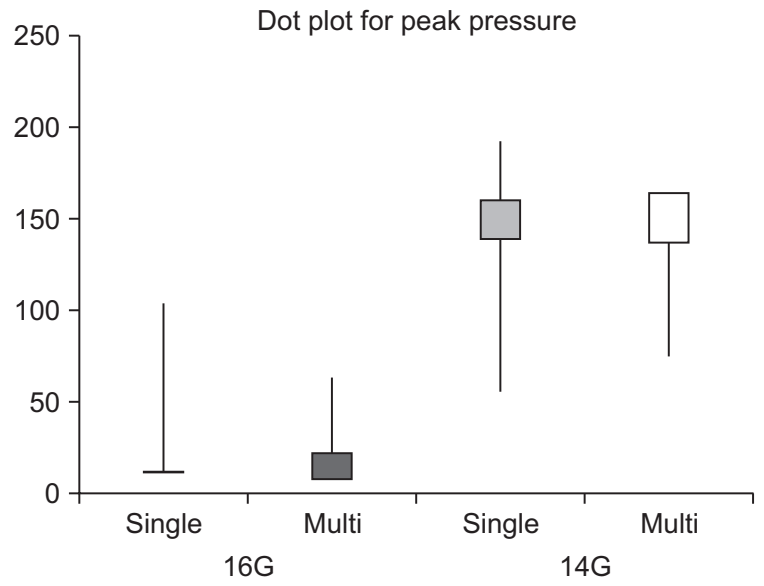

Fig. 4. A dot plot for visual comparison. $\mathrm{G}$, gauge. stepwise multistep ablation with $14 \mathrm{G}$ antenna, $106 \pm 43.8 \mathrm{~mm} \mathrm{Hg}$. Standard single-step ablation with a $14 \mathrm{G}$ antenna resulted in the highest peak pressure among the groups.

We measured the rise of intrahepatic pressure clearly during MWA, although explosive sounds was not heard during MWA procedures. We found that hepatic parenchymal pressure increased more in groups treated with a $14 \mathrm{G}$ antenna than those treated with a 16G antenna in both single and stepwise treatments. There were no statistically significant differences in the rise of pressure between single-step ablation and stepwise ablation if the antenna gauge remained constant (Kruskal Wallis test and Tukey test using ranks, $\mathrm{p}>0.05$ ) (Table 4).

\section{Ablation volume}

The average value of ablation volume was as follows: standard single-step ablation with $16 \mathrm{G}$ antenna, $4.63 \pm 0.5 \mathrm{~cm}^{3}$; stepwise multistep ablation with $16 \mathrm{G}$ antenna, $3.75 \pm 0.8 \mathrm{~cm}^{3}$; standard single-step ablation with $14 \mathrm{G}$ antenna, $15.33 \pm 3.4 \mathrm{~cm}^{3}$; stepwise multistep ablation with $14 \mathrm{G}$ antenna, $10.98 \pm 2.5 \mathrm{~cm}^{3}$. The ablation areas in each groups appeared oval or spherical in shape (Fig. 5). The ablation volume of groups treated with a $14 \mathrm{G}$ antenna was larger than that of groups treated with a $16 \mathrm{G}$ antenna, and the ablation volume of single-step groups was larger than that of stepwise groups in each gauge. There was statistical difference in ablation volume between single-step ablation and stepwise multistep ablation in the $14 \mathrm{G}$ groups. There was also a statistical difference in ablation volume between $14 \mathrm{G}$ and $16 \mathrm{G}$ antennae in single and stepwise groups. However, there was no statistical difference in ablation volume between single-step ablation and stepwise ablation in the 16G group (Kruskal Wallis test and Tukey test using ranks, $\mathrm{p}<0.05$ ) (Table 5).

\section{Ablation time}

The ablation time was as follows: standard single-step ablation with $16 \mathrm{G}$ antenna, 675 seconds; stepwise multistep ablation with 16G antenna, 401 seconds; standard single-step ablation with $14 \mathrm{G}$ antenna, 339 seconds; stepwise multistep ablation with $14 \mathrm{G}$ antenna, 306 seconds. The reason why there was a

Table 4. Comparison of Intrahepatic Pressure for Four Groups (Tukey Test Using Ranks)

\begin{tabular}{lcccc}
\hline & $14 \mathrm{G}$ single & 16G single & 14G multi & 16G multi \\
\hline $14 \mathrm{G}$ single & - & - & - & - \\
$16 \mathrm{G}$ single & $<0.0005^{*}$ & - & - & - \\
$14 \mathrm{G}$ multi & $0.9647^{\dagger}$ & 0.0018 & - & - \\
$16 \mathrm{G}$ multi & 0.0003 & $0.9967^{\dagger}$ & $0.0010^{\ddagger}$ & - \\
\hline
\end{tabular}

*Maximum pressures of single 14-gauge (G) group and single 16G group were significantly different; ${ }^{\dagger}$ There were no significant differences in the rise of pressure between single-step ablation and stepwise ablation within the same gauge groups; ${ }^{\ddagger}$ Maximum pressures of stepwise $14 \mathrm{G}$ group and stepwise $16 \mathrm{G}$ group were significantly different. 


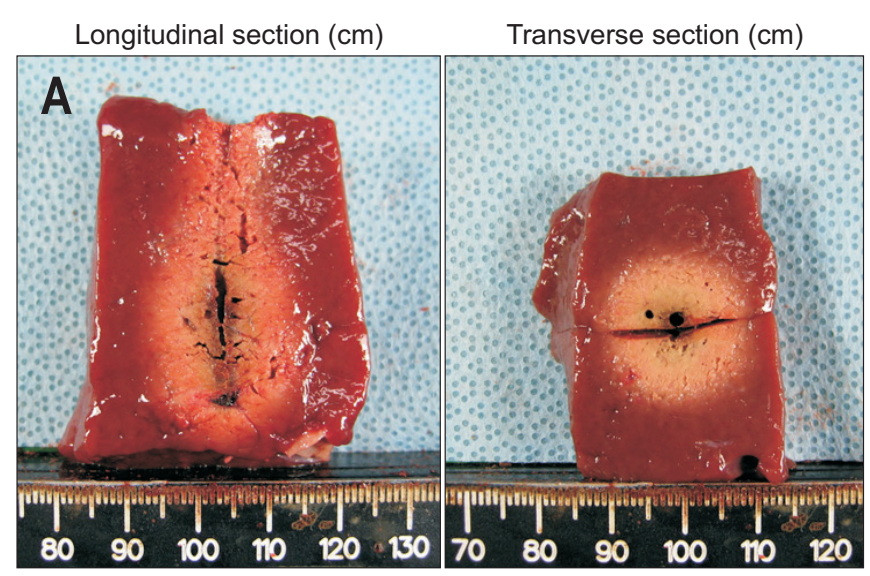

$1.60 \times 3.05$
$1.66 \times 1.74$

Transverse section $(\mathrm{cm})$

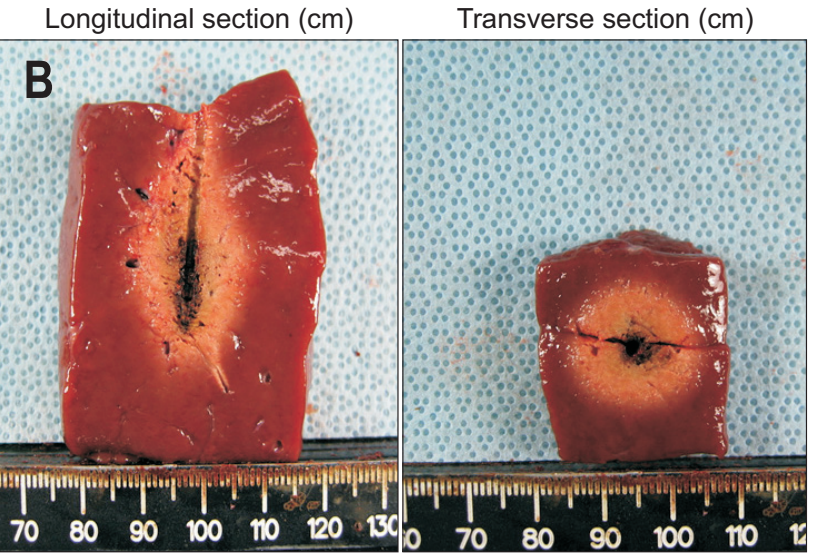

$1.75 \times 2.89$

$1.55 \times 1.73$

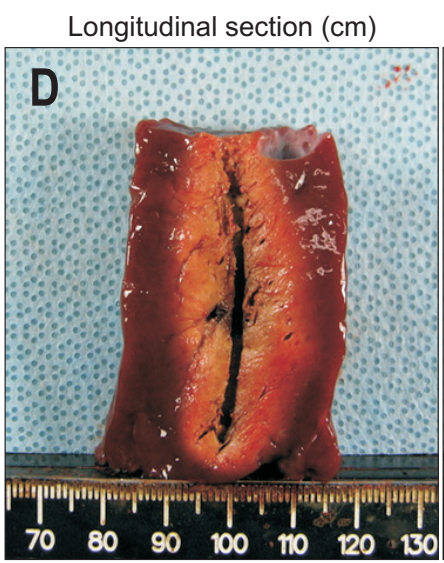

$1.95 \times 4.34$
Transverse section $(\mathrm{cm})$

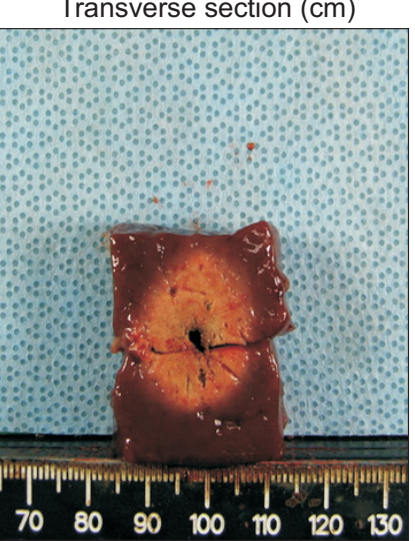

$1.92 \times 2.14$

$1.75 \times 2.89$

$1.55 \times 1.73$

Fig. 5. Cross-sectional image. The depth of a longitudinal section and the longest and shortest axes (means) of the ablation area were measured. The ablation areas appeared uniformly oval in four groups: (A) Cross-section of ablation zone by single-step ablation with a 16-gauge (G) antenna. (B) Cross-section of ablation zone by multistep ablation with a 16G antenna. (C) Cross-section of ablation zone by single-step ablation with a $14 \mathrm{G}$ antenna. (D) Cross-section of ablation zone by multistep ablation with a $14 \mathrm{G}$ antenna.

Table 5. Comparison of Ablation Volume for Four Groups (Tukey Test Using Ranks)

\begin{tabular}{lcccc}
\hline & $14 \mathrm{G}$ single & $16 \mathrm{G}$ single & $14 \mathrm{G}$ multi & $16 \mathrm{G}$ multi \\
\hline $14 \mathrm{G}$ single & - & - & - & - \\
$16 \mathrm{G}$ single & $<0.0001^{*}$ & - & - & - \\
$14 \mathrm{G}$ multi & $0.0243^{\dagger}$ & $<0.0001$ & - & - \\
$16 \mathrm{G}$ multi & $<0.0001$ & $0.0682^{\ddagger}$ & $<0.0001^{\S}$ & - \\
\hline
\end{tabular}

*Ablation volumes of single 14-gauge (G) group and single $16 \mathrm{G}$ group were significantly different; ${ }^{\dagger}$ Ablation volumes of single $14 \mathrm{G}$ group and stepwise $14 \mathrm{G}$ group were significantly different; ${ }^{\ddagger} \mathrm{Abla}-$ tion volumes of single $16 \mathrm{G}$ group and stepwise $16 \mathrm{G}$ group were not significantly different; ${ }^{\S}$ Ablation volumes of stepwise $14 \mathrm{G}$ group and stepwise $16 \mathrm{G}$ group were significantly different.

difference in running time was that microwave generator was stopped for a while and was given repeatedly when microwave rises more than target watt, so MWA was applied intermittently.

\section{DISCUSSION}

RFA is becoming an accepted treatment modality for many tumors of the liver and is being explored for tumors in the lung, kidney, and bone. ${ }^{1}$ While RF energy is the most familiar heat source for tissue ablation, it has several limitations. ${ }^{2}$ Kotoh et al. ${ }^{3}$ and Seki et al. ${ }^{9}$ reported rapid and scattered recurrences and suggested that increased intratumoral pressure might be the cause. ${ }^{19}$ Explosive sounds like a "pop" are often heard during RFA procedures, and microbubbles are frequently observed by ultrasonography. ${ }^{20}$ During exposure to a high-frequency current, the tumor is heated and generates air bubbles throughout the treatment period. ${ }^{21}$ These bubbles enter the blood vessels, and rapid tumor heating may lead to unpredicted increases in intrahepatic pressure. $^{22}$

Microwave energy may be a better source for tissue ablation. ${ }^{13}$ A MWA is essentially induced dielectric heating using oscillation of water molecules. ${ }^{23}$ When an oscillating electric charge from radiation interacts with a water molecule, it causes the molecule to flip.

The technique may be useful for flexible approaches to treatment, including percutaneous, laparoscopic, and open surgical approximation. With imaging guidance, the tumor is localized and a thin microwave antenna is placed directly into the target 
lesion. ${ }^{13,15-18,23-27}$ A microwave generator emits an electromagnetic wave through the exposed, noninsulated portion of the antenna. Electromagnetic microwaves induce water molecules in the surrounding tissue, producing friction and heat and causing cellular death via coagulation necrosis. ${ }^{26}$ MWA has promising potential in the treatment of primary and secondary liver disease, primary and secondary lung malignancies, renal and adrenal tumors, and bone metastases. ${ }^{15}$

The major difference between MWA and RFA is that MWA occurs in a volume around the applicator antenna, while RFA is restricted to areas of high current density. RFA requires an electrically conductive path, while microwaves do not; thus, microwaves are capable of propagating through materials with low or zero conductivity. This means that low conductivity tissues inhibit RF current flow, but allow better microwave propagation. One important factor when comparing RFA and MWA is the tumor's host organ and location within the organ, since the tissue properties of normal liver, lung, kidney, and bone are quite different. ${ }^{13,26}$

Intratumoral pressure is expected to rise during ablation, due to the heating of the tissue. No previous studies have investigated intratumoral pressure during MWA. Kotoh et al. ${ }^{10}$ recently reported an evaluation of liver parenchymal pressure during RFA in an in vivo porcine model.

Our results showed that increased intrahepatic pressure is evident during MWA, which was observed when both 16G and $14 \mathrm{G}$ antenna were used; however, the increase in pressure was much more prominent when the $14 \mathrm{G}$ antenna was used, regardless of the single-step versus multistep protocol. Multistep protocols produced a slightly smaller intrahepatic pressure rise than single-step protocols, but this was not statistically significant. We hypothesize that MWA, especially when used in a multistep protocol, produced less pressures increases. This might be due to differences in the lower ablation time and even distribution of the MWA (Table 2).

The present experiment found that the size and the volume of ablation zone tended to be small when compared with the general RFA experiment. Sixteen-gauge antennas created smaller zones of ablation than $14 \mathrm{G}$ antennas, and multistep protocols created smaller ablation zones than single-step protocols (Table 2). The reason why smaller ablation volume when using $16 \mathrm{G}$ antennas or multistep protocols compared with $14 \mathrm{G}$ antennas or single-step protocols was water vaporization process due to the high heat generation from MWA due to more increased intrahepatic pressure at latter conditions. These results may originate from the low power protocol (902 to $928 \mathrm{MHz}$ ) generator used in our experiment. ${ }^{28}$ However, there was no statistical difference in ablation volume between the multistep groups and singlestep groups using a $14 \mathrm{G}$ antenna.

And actual temperature of cow liver tissue was inappropriate due to indirect method for warming. Even ex vivo study at room temperature produce ablation zone larger compared to in vivo. Thus, preheating the tissue is not warranted as it will further amplify the ablation zone compared to in vivo.

In this study, we found that varying settings of the MWA device could influence the pressure during ablation. The multistep method required a slightly lower pressure during ablation than the single-step method. That is, the single-step procedure requires continuous ablation until the entire targeted region becomes necrotic. In contrast, in the multistep procedure, the region of ablation is confined in each step.

There were several limitations to this study that have to be acknowledged. The first limitation concerns the bovine liver model. These livers were pure parenchyma and contained no tumor, and it is likely that there are differences between normal liver and tumor. As we used ex vivo bovine liver, we found it difficult to maintain body temperature because vapor produced during MWA leaked through vessels or fissure, exposing the cut surface of the liver. Therefore, a further in vivo study will be necessary. Second, we were also limited by the small sample size of each group, in which only 10 pieces of cow liver were used. If larger samples were used, more results may have been statistically significant. Third, we only used peak pressure measurement. Tracking the changes in pressure over time might reveal more about how the gas bubbles form. Third, the pressure was measured while the bovine liver was in open space, whereas previous ex vivo study used a sealed box. ${ }^{11}$ Finally, this study was limited by the use of technology still in development. RFA devices are evolving rapidly, and the MWA device used in this study are undergoing continued development. In particular, the MWA generator used in this experiment does not have a large ablation zone due to a low power protocol (902 to $928 \mathrm{MHz}$ ). ${ }^{28}$ Therefore, more research is needed to assess its mechanism of action in a more powerful MWA device.

In this study, we showed that the standard single-step method might entail a risk of an extreme increase in intratumoral pressure under some conditions, which could result in intrahepatic metastasis or rapid progression after the procedure. However, intratumoral pressure can be reduced by adopting an incremental, multistep method during MWA. We believe that this multistep method might be useful as a standard clinical procedure for MWA, after optimization of the MWA power.

In conclusion, MWA resulted in increases in intrahepatic pressure. Single-step methods resulted in larger pressure increases than multistep methods. Unfortunately, increased intrahepatic pressure could not be controlled using multistep methods. Further study is needed to prove that multistep methods may be able to be used to prevent increased intrahepatic pressure during MWA after applying the proper power.

\section{CONFLICTS OF INTEREST}

No potential conflict of interest relevant to this article was reported. 


\section{ACKNOWLEDGEMENTS}

This study was supported by Samsung Medical Center grant (\#GF01130071) and Samsung Biomedical Research Institute grant (\#CB11261).

\section{REFERENCES}

1. Rossi S, Garbagnati F, Rosa L, Azzaretti A, Belloni G, Quaretti P. Radiofrequency thermal ablation for treatment of hepatocellular carcinoma. Int J Clin Oncol 2002;7:225-235.

2. Tateishi R, Shiina S, Teratani T, et al. Percutaneous radiofrequency ablation for hepatocellular carcinoma: an analysis of 1000 cases. Cancer 2005;103:1201-1209.

3. Kotoh K, Enjoji M, Arimura E, et al. Scattered and rapid intrahepatic recurrences after radio frequency ablation for hepatocellular carcinoma. World J Gastroenterol 2005;11:6828-6832.

4. Nicoli N, Casaril A, Abu Hilal M, et al. A case of rapid intrahepatic dissemination of hepatocellular carcinoma after radiofrequency thermal ablation. Am J Surg 2004;188:165-167.

5. Shirato K, Morimoto M, Tomita N, et al. Hepatocellular carcinoma: a case of extrahepatic seeding after percutaneous radiofrequency ablation using an expandable needle electrode. Hepatogastroenterology 2002;49:897-899.

6. Takada Y, Kurata M, Ohkohchi N. Rapid and aggressive recurrence accompanied by portal tumor thrombus after radiofrequency ablation for hepatocellular carcinoma. Int J Clin Oncol 2003;8:332335.

7. Vergara Gómez M, Miquel Planas M, Gil Prades M, et al. Rapid progression of hepatocellular carcinoma after surgery: apropos of a case and review of the literature. Gastroenterol Hepatol 2010;33:569-573.

8. Portolani N, Tiberio GA, Ronconi M, et al. Aggressive recurrence after radiofrequency ablation of liver neoplasms. Hepatogastroenterology 2003;50:2179-2184.

9. Seki T, Tamai T, Ikeda K, et al. Rapid progression of hepatocellular carcinoma after transcatheter arterial chemoembolization and percutaneous radiofrequency ablation in the primary tumour region. Eur J Gastroenterol Hepatol 2001;13:291-294.

10. Kotoh K, Morizono S, Kohjima M, Enjoji M, Sakai H, Nakamuta M. Evaluation of liver parenchymal pressure and portal endothelium damage during radio frequency ablation in an in vivo porcine model. Liver Int 2005;25:1217-1223.

11. Kotoh K, Nakamuta M, Morizono S, et al. A multi-step, incremental expansion method for radio frequency ablation: optimization of the procedure to prevent increases in intra-tumor pressure and to reduce the ablation time. Liver Int 2005;25:542-547.

12. Kawamoto C, Yamauchi A, Baba Y, Kaneko K, Yakabi K. Measurement of intrahepatic pressure during radiofrequency ablation in porcine liver. J Gastroenterol 2010;45:435-442.

13. Brace CL. Radiofrequency and microwave ablation of the liver, lung, kidney, and bone: what are the differences? Curr Probl Diagn Radiol 2009;38:135-143.

14. Shiozawa K, Watanabe M, Takahashi M, Wakui N, Iida K, Sumino Y. Analysis of patients with rapid aggressive tumor progression of hepatocellular carcinoma after percutaneous radiofrequency ablation. Hepatogastroenterology 2009;56:1689-1695.

15. Simon CJ, Dupuy DE, Mayo-Smith WW. Microwave ablation: principles and applications. Radiographics 2005;25 Suppl 1:S69S83.

16. Laeseke PF, Lee FT Jr, Sampson LA, van der Weide DW, Brace CL. Microwave ablation versus radiofrequency ablation in the kidney: high-power triaxial antennas create larger ablation zones than similarly sized internally cooled electrodes. J Vasc Interv Radiol 2009;20:1224-1229.

17. Knavel EM, Hinshaw JL, Lubner MG, et al. High-powered gascooled microwave ablation: shaft cooling creates an effective stick function without altering the ablation zone. AJR Am J Roentgenol 2012;198:W260-W265.

18. Lubner MG, Brace CL, Hinshaw JL, Lee FT Jr. Microwave tumor ablation: mechanism of action, clinical results, and devices. J Vasc Interv Radiol 2010;21:S192-S203.

19. Eick OJ, Gerritse B, Schumacher B. Popping phenomena in temperature-controlled radiofrequency ablation: when and why do they occur? Pacing Clin Electrophysiol 2000;23:253-258.

20. Livraghi T, Goldberg SN, Lazzaroni S, et al. Hepatocellular carcinoma: radio-frequency ablation of medium and large lesions. Radiology 2000;214:761-768.

21. Iida H, Aihara T, Ikuta S, Yamanaka N. Effectiveness of impedance monitoring during radiofrequency ablation for predicting popping. World J Gastroenterol 2012;18:5870-5878.

22. Fernandes ML, Lin CC, Lin CJ, Chen WT, Lin SM. Prospective study of a 'popping' sound during percutaneous radiofrequency ablation for hepatocellular carcinoma. J Vasc Interv Radiol 2010;21:237-244.

23. Brace CL. Microwave ablation technology: what every user should know. Curr Probl Diagn Radiol 2009;38:61-67.

24. Bui JT, Gaba RC, Knuttinen MG, et al. Microwave lung ablation complicated by bronchocutaneous fistula: case report and literature review. Semin Intervent Radiol 2011;28:152-155.

25. de Baere T. New techniques of tumor ablation (microwaves, electroporation). J Radiol 2011;92:789-795.

26. Dupuy DE. Microwave ablation compared with radiofrequency ablation in lung tissue-is microwave not just for popcorn anymore? Radiology 2009;251:617-618.

27. Jiao DC, Zhou Q, Han XW, et al. Microwave ablation treatment of liver cancer with a 2,450-MHz cooled-shaft antenna: pilot study on safety and efficacy. Asian Pac J Cancer Prev 2012;13:737-742.

28. Hoffmann R, Rempp H, Erhard L, et al. Comparison of four microwave ablation devices: an experimental study in ex vivo bovine liver. Radiology 2013;268:89-97. 